\title{
IMMUNOLOGICAL NON-RESPONSIVENESS AND ACQUISITION OF TOLERANCE IN RELATION TO IMMUNE PRIVILEGE IN THE EYE
}

\author{
J. WAYNE STREILEIN \\ Boston, Massachusetts
}

\begin{abstract}
SUMMARY
Immune privilege is a dynamic, physiological process that enables the eye to accept foreign tissue grafts in an unprecedented fashion. Privilege is actively acquired and maintained by immune regulatory forces that represent an important form of antigen-specific immunological tolerance. Privilege in the eye results from eye-dependent modifications in the induction (afferent limb) and expression (efferent limb) of immunity to intraocular antigens. The eye-dependent features that are important in privilege include integrity of the blood-ocular barrier, the virtual absence of lymphatics, an afferent drainage pathway that is almost exclusively via the blood vasculature, and an immunosuppressive intraocular microenvironment. This microenvironment is comprised of a variety of cytokines and neuropeptides that (1) impair antigen-driven activation of primed and alloreactive $T$ cells, (2) suppress effector functions of activated macrophages, and (3) modify the antigen processing and presenting properties of indigenous, bone-marrow-derived professional antigen presenting cells. Eye-derived antigenic signals, which escape when local antigen presenting cells migrate via the blood to the spleen, selectively activate regulatory $T$ cells that impair the development of antigen-specific delayed hypersensitivity and complement fixing antibodies, a phenomenon termed anterior chamber associated immune deviation (ACAID). ACAID has been implicated in the extraordinary success of orthotopic corneal allografts, as well as the prolonged intraocular survival enjoyed by transplants of retinal tissues. The active features of immune privilege can be exploited to secure successful corneal and retinal transplantation.
\end{abstract}

Susceptibility to immune rejection represents a major barrier to the success of intraocular transplants of allogeneic and xenogeneic tissue. As in other

Correspondence to: J. Wayne Streilein MD, Schepens Eye Research Institute, Harvard Medical School, 20 Staniford Street, Boston, MA 02114, USA. essential tissue grafts, prevention of graft rejection by creating a state of specific non-responsiveness or tolerance is a major goal of basic research. In the special case of the eye, the phenomenon of immunological privilege has been demonstrated experimentally. ${ }^{1,2}$ Because of the existence of privilege, it has been anticipated that susceptibility of ocular grafts to rejection would be reduced. In this review, immune privilege in the eye is defined and described in contemporary terms, and strategies for improving intraocular graft survival are considered, based on an understanding of the physiological mechanisms responsible for immune privilege.

Ocular immune privilege can be defined operationally as follows: foreign tissues placed in the anterior chamber, the vitreous cavity, the subretinal space and the corneal stroma experience extended (even indefinite) survival compared with similar tissues placed subcutaneously (a conventional site).

Studies in the 1940s and 1950s revealed the internal compartments of the eye to lack a demonstrable lymphatic drainage, and it was proposed and accepted at the time that immune privilege could be explained by an 'afferent blockade' in which the systemic immune apparatus never became aware of or recognised antigenic materials placed intraocularly. ${ }^{1}$ More recent studies have confirmed that, under normal circumstances, lymphatic drainage from the internal compartments of the eye is minimal. However, immunologists now appreciate that antigenic material also escapes from the eye through the aqueous humour outflow tract. Moreover, it has recently been determined that prolonged intraocular graft survival is sustained by active immune regulatory mechanisms, rather than by passive ignorance of the graft's existence., ${ }^{2,3}$

As experimentalists have expanded studies of the fate of tissue grafts placed within the eye, it has become clear that immune privilege is not 'absolute'. This means that not all foreign tissues placed in the 
eye enjoy indefinite survival; in many instances, a 'honeymoon' period of prolonged acceptance is followed by the emergence of destructive immunity whereupon the graft is destroyed. Since immune privilege in the eye is actively acquired and maintained, hope has arisen that therapeutic strategies can be devised that would significantly modify graft survival - in positive or negative directions.

On the basis of studies carried out in the midtwentieth century, investigators believed that immune privilege resulted solely from a defect in the process by which antigenic information first reaches the immune system, i.e. a defect in the afferent limb of immune induction was postulated. ${ }^{1}$ Since in many instances systemic immunisation curtailed the survival of intraocular grafts, it was deduced that expression of systemic immunity is 'normal' in the eye, i.e. there was believed to be no defect in the efferent limb of the immune response. However, more critical studies carried out in the last decade have revealed that expression of immunity in the eye is by no means 'normal' or complete. Particularly, it has been reported that expression of cell-mediated immunity, dependent upon $\mathrm{T}$ cells of the delayed hypersensitivity type, is grossly impaired in the eye, ${ }^{4}$ and there is evidence that complement activation is also reduced or absent within the anterior chamber. Thus, the contemporary view is that ocular immune privilege is multifactorial in origin and results from alterations in both induction and expression of immunity to antigens on tissue grafts placed within the eye.

\section{OCULAR IMMUNE PRIVILEGE: A RATIONALE}

As immune privilege in the eye has been found to have a major modifying influence on intraocular graft survival and in other ocular inflammatory disorders, investigators have begun to inquire into the reason for the existence of immune privilege - in the eye, as well as at other sites in the body where privilege is also found (e.g. brain, testis). At present, the dominant view is that immune privilege in the eye represents an evolutionary adaptation that is designed to limit intraocular expression of immunogenic inflammation. Blindness is a powerful selective force, and since immunogenic inflammation within the eye is extremely deleterious to vision, selection has limited expression of this type of immunity within the eye.

Ocular immune privilege has been studied in a variety of experimental situations. The phenomenon is now implicated in (1) the extraordinary success of orthotopic corneal allografts, and intraocular retinal cell and tissue transplants, ${ }^{5,6}$ (2) the unfortunate success of progressively growing intraocular tumours, $^{7}$ (3) the pathogenesis of stromal keratitis secondary to infection with herpes simplex virus, ${ }^{8}$ (4) the development of acute retinal necrosis following intraocular infection with herpes viruses, ${ }^{9}$ and (5) protection against, and amelioration of, experimental autoimmune uveoretinis. ${ }^{10}$

\section{FEATURES OF THE EYE PERTINENT TO IMMUNE PRIVILEGE}

There are biochemical and anatomical features of the eye that appear to be critical to the existence of immune privilege. First, the barrier that exists between the blood and the internal compartments of the eye is extremely important. This barrier, which severely restricts the inflow of blood-borne molecules and cells into the eye, largely prevents specific and non-specific mediators of immunogenic inflammation from gaining access to the eye. Second, the relative lack of lymphatic drainage combined with the bulk flow of aqueous humour through the trabecular meshwork into the canal of Schlemm (and thus into the venous circulation) ensures that antigenic material that escapes the eye does so almost exclusively by the blood route. Consequently, the spleen rather than draining lymph nodes acts as the primary lymphoid organ for the receipt and transduction of antigenic signals. Third, bone-marrowderived cells with morphological and surface phenotypic characteristics typical of professional antigen presenting cells (APC) are present within the tissues of the eye: iris, ciliary body, retina. ${ }^{11,12}$ However, the functional properties of these cells are distinctly atypical compared with their cellular counterparts at conventional body sites, and the type of immunity evoked by antigens presented by these cells is 'deviant'. ${ }^{3}$ Fourth, ocular fluids contain a unique spectrum of factors (cytokines, neuropetides, growth factors) that suppress immunogenic inflammation and complement activation. ${ }^{14}$ To date, aqueous humour has been found to contain physiologically relevant concentrations of transforming growth factor-beta (TGF $\beta)$, alpha-melanocyte stimulating hormone, vasoactive intestinal peptide, calcitonin gene-related peptide, and 'anti-complementary activity' that has yet to be defined chemically. Moreover, aqueous humour is grossly deficient in cortisol binding globulin; ${ }^{15}$ since the concentration of cortisol in aqueous humour approaches that of blood plasma, the 'effective' pharmacological concentration of cortisol in this fluid is quite high, and this probably contributes to the fluid's immunosuppressive features.

Taken together, these anatomical and biochemical features contribute to the immune privilege that is extended to foreign tissue grafts placed within the eye. It is likely that experimentalists will identify additional features in the future that make important contributions as well. 


\section{ANTERIOR CHAMBER ASSOCIATED IMMUNE DEVIATION: ITS ROLE IN OCULAR IMMUNE PRIVILEGE}

The modern notion that immune privilege is actively, rather than passively, maintained was initiated approximately 20 years ago when Kaplan and Streilein demonstrated that rats pretreated with allogeneic lymphoid cells in the anterior chamber (AC) accepted for a prolonged interval of time orthotopic skin allografts syngeneic with the ACinjected cells. ${ }^{16}$ Since then, a wide variety of antigens has been injected into the AC of laboratory rats and mice in order to assess the subsequent systemic immune response. In general, a stereotypic pattern of immunity is elicited, now termed Anterior Chamber Associated Immune Deviation (ACAID). ${ }^{2,3}$ Based on these experimental data, ACAID is defined operationally as follows: antigen placed in the anterior chamber evokes a selective, systemic immune deficiency in which the missing elements are $\mathrm{T}$ cells that mediate delayed hypersensitivity and $B$ cells that secrete complement fixing antibodies. At the same time, other immune effector modalities are generated, including primed precursor cytotoxic $\mathrm{T}$ cells in lymph nodes and spleen, and non-complement fixing IgG antibodies in the serum.

ACAID has been characterised as distinctive from conventional immune responses in a number of important ways. First, except for highly immunogenic antigens (class I MHC molecules, tumour antigens induced by ultraviolet radiation), ACAID in response to AC-injected antigens is extremely long-lasting. Experimentally, ACAID has been demonstrated to persist in mice for at least 6 months. ${ }^{17}$ Second, when antigen is injected simultaneously into the $\mathrm{AC}$ and into the subcutaneous space, ACAID is routinely induced, rather than conventional immunity. This indicates that ACAID is dominant to conventional immunity, and strongly suggests that both are highly regulated. Third, injection of antigen into the $\mathrm{AC}$ of mice presensitised conventionally to the same antigen also results in ACAID, indicating that the regulatory mechanisms of ACAID can even suppress preformed memory and effector $\mathrm{T}$ cells that mediate delayed hypersensitivity. ${ }^{18}$ Fourth, a systemic response identical to ACAID is evoked when antigens are placed in the vitreous cavity or in the subretinal space. ${ }^{19}$ Thus, immune deviation of the AC type appears to be a property of all three ocular compartments. Fifth, ACAID has now been demonstrated following antigen injections into the $\mathrm{AC}$ of eyes of mice, rats, and primates (Cynomolgus). ${ }^{20}$

\section{CELLULAR MECHANISM OF ACAID INDUCTION}

Two provocative observations have led to an under- standing of the cellular mechanisms by which ACAID is induced. On the one hand, enucleation of the antigen-containing eye (even as late as 5 days after AC injection) aborts ACAID. On the other hand, removal of the spleen - prior to AC injection of antigen, or as late as 5 days thereafter - also prevents ACAID induction. These findings suggest that antigenic signals must escape the eye and be received by the spleen during ACAID induction. In examining this point, recent experiments have revealed that if blood is assayed within 48 hours of AC injection of an antigen, an ACAID-inducing signal is present. ${ }^{21}$ Whether soluble antigens (such as bovine serum albumin or interphotoreceptor retinol binding protein), or alloantigens on lymphoreticular cells are injected into the $\mathrm{AC}$, the blood eventually contains a monocyte/dendritic cell (F4/ $80+)$ that induces ACAID when injected intravenously into naive, syngeneic mice. When such cells are injected subcutaneously, conventional immunity is induced. Cells with similar ACAID-inducing capability can be harvested directly from iris and ciliary bodies of eyes that received an AC injection of antigen 24 hours previously, and F4/80+ cells from iris and ciliary body of normal eyes that are pulsed in vitro with soluble antigen induce ACAID when injected intravenously into naive mice. In fact, F4/ $80+$ cells from the blood or periteonal cavity (which possess no constitutive ACAID-inducing properties) induce ACAID if pulsed with antigen in vitro and injected into the AC of normal mouse eyes. These results implicate the microenvironment of the anterior chamber in conferring ACAID-inducing properties on conventional antigen presenting cells. This has been demonstrated directly. Conventional APC that are pulsed with antigen in vitro in the presence of TGF $\beta$ (or fluids containing this cytokine) acquire ACAID-inducing potential. When such cells are injected intravenously into naive mice, the latter fail to develop antigen-specific delayed hypersensitivity when immunised conventionally with antigen in adjuvant. ${ }^{22}$

APC that have been exposed to TGF $\beta$ in vitro display unique migration properties when injected intravenously. These cells migrate only to the spleen - not to other lymphoid organs. ${ }^{23}$ Their ultimate location in the spleen has yet to be determined, but it is likely that they come to rest in an environment containing $\mathrm{T}$ lymphocytes. This conclusion has been reached from studies designed to determine whether ACAID-inducing cells are actually functioning as APC. Two types of experiments bear on this issue. ${ }^{24,25}$ In the first, conventional APC have been incubated with soluble protein antigen (bovine serum albumin, ovalbumin) in the presence of TGF $\beta$; and during this interval, the cells were exposed to the drug chloroquine. This agent 
prevents acidification of endosomal vesicles and thereby prevents the cleavage of endocytosed protein antigens into immunogenic peptide fragments. Whereas antigen pulsed cells treated with TGF $\beta$ induced ACAID when injected intravenously, similar cells also exposed to chloroquine failed to induce ACAID. This outcome strongly suggests that in order for cells to promote ACAID they must be able to endocytose soluble protein into vesicles that become acidified. In the second set of experiments, in-vitro-generated ACAID-inducing cells have been injected into allogenic mice selected to determine whether major or minor transplantation antigens act as restricting elements for ACAID induction. The results indicate that in order for ACAID to emerge, the donor and recipient of ACAID-inducing cells must share at least one class I MHC or one class II MHC alloantigen. Donor-recipient pairs that merely share minor histocompatibility antigens do not permit ACAID to develop. Taken together, these results support the view that cells with ACAIDinducing capability actually function as APC to selfMHC-restricted T cells.

Experiments of this type have been conducted with transgenic mice in which the gene for beta-2 microglobulin $(\beta 2 \mathrm{~m})$ has been deleted. Cells of mice of this type express few, if any, class I MHC molecules. It has now been reported that (1) ACAID cannot be induced by AC injection of soluble antigen into the $\mathrm{AC}$ of $\beta 2 \mathrm{~m}$-deficient mice, and (2) conventional APC obtained from $\beta 2 \mathrm{~m}$ deficient mice fail to acquire ACAID-inducing properties when pulsed with antigen and TGF $\beta$ in vitro. Although a completely satisfying explanation for these results has yet to emerge, the findings emphasise the paramount importance of class I MHC molecules in the genesis of ACAID.

To summarise, immune privilege depends in part upon the induction of ACAID which occurs when antigen is placed within any of several intraocular compartments. ACAID develops because intraocular APC, under the influence of local immunomodulatory factors (chiefly $\mathrm{TGF} \beta$ ), capture intraocular antigenic material and migrate with it via the blood to the spleen. At this tissue site, these 'deviant' APC process eye-derived antigens in a unique fashion which enables them to present to, as well as to activate, distinctive regulatory $\mathrm{T}$ cells. Importantly, APC bearing eye-derived antigens do not activate delayed hypersensitivity $\mathrm{T}$ cells.

\section{ACAID AND OCULAR ALLOGRAFTS}

Our laboratory has begun to explore and define the extent to which ACAID participates when immune privilege is extended to ocular allografts in mice and rats. To summarise the most recent work: (1) AC injections of allogeneic lymphoreticular cells (spleen, lymph node, peritoneal exudate cells), or intravenous injections of allogeneic lymphoid cells incubated in vitro with TGF $\beta$ induce ACAID. ${ }^{26}$ Moreover, both major and minor histocompatibility antigens can induce the phenomenon. (2) AC or vitreous cavity injections of allogenic tumour cells induce ACAID that is permanent (if tumours express only minor histocompatibility antigens) or transient (if tumours express MHC class I alloantigens). ${ }^{27}$ (3) Longstanding, healthy allogeneic corneas grafted orthotopically into normal mouse eyes are associated with donor-specific ACAID, whereas mice with irreversibly rejected corneal grafts display no ACAID. ${ }^{5}$ (4) Pre-emptive induction of ACAID by donor alloantigens fails to prevent acute rejection of corneal allografts placed in normal mouse eyes (unpublished observations). Since the mice still display ACAID after the corneal graft has succumbed to rejection, the effector mechanism responsible for rejection must not be $\mathrm{T}$ cells that mediate delayed hypersensitivity. (5) Prolonged intraocular acceptance of allogeneic grafts of neonatal neuronal retina, as well as retinal pigment epithelium, is associated with ACAID. ${ }^{19,28}$ Moreover, if and when these grafts are destroyed immunologically, ACAID disappears and delayed hypersentivity emerges.

We believe that immune privilege and ACAID are important eye-specific processes that protect the eye from the binding effects of immunogenic inflammation. Moreover, we believe that these processes play an important role in acceptance of allogeneic tissue grafts designed to restore sight to blinded eyes. However, it must be recalled that the immune system possesses a wide array of effector mechanisms with which solid tissue allografts can be destroyed. Thus, since ACAID appears to interfere only with delayed hypersensitivity and complement fixing antibody expression, grafts placed within the eye of animals with ACAID are nonetheless vulnerable to other destructive effector modalities, such as cytotoxic $\mathrm{T}$ cells and non-complement fixing antibodies. Our goal is to identify those forms of intraocular graft rejection that are mediated solely by delayed hypersensitivity, and in these forms to attempt to invoke the protective effects of ACAID.

Key words: Anterior chamber associated immune deviation, Delayed hypersensitivity, Immune privilege, Immunosuppressive microenvironment.

\section{REFERENCES}

1. Medawar P. Immunity to homologous grafted skin. III. The fate of skin homografts transplanted to the brain, to subcutaneous tissue, and to the anterior chamber of the eye. Br J Exp Pathol 1948;29:58-69.

2. Niederkorn JY. Immune privilege and immune regulation in the eye. Adv Immunol 1990;48:191-226.

3. Streilein JW. Immune regulation and the eye: a dangerous compromise. FASEB J 1987;1:199-208.

4. Cousins S, Trattler W, Streilein JW. Immune privilege 
and suppression of immunogenic inflammation in the anterior chamber of the eye. Curr Eye Res 1991; 10:287-97.

5. Sonoda Y, Streilein JW. Impaired cell mediated immunity in mice bearing healthy orthotopic corneal allografts. J Immunol 1993;150:1727-34.

6. Jiang LQ, Streilein JW. Immunologic privilege evoked by histocompatible intracameral retinal transplants. Regional Immunol 1991;3:121-30.

7. Niederkorn J, Streilein JW, Shadduck JA. Deviant immune responses to allogeneic tumours injected intracamerally and subcutaneously in mice. Invest Ophthalmol Vis Sci 1980;20:355-63.

8. McLeish W, Rubsamen P, Atherton SS, Streilein JW. Immunobiology of Langerhans cells on the ocular surface. II. Role of central corneal Langerhans cells in stromal keratitis following experimental HSV-1 infection in mice. Regional Immunol 1989;2:236-43.

9. Streilein JW, Atherton S, Vann VA. Critical role for ACAID in the distinctive pattern of retinitis that follows anterior chamber inoculation of HSV-1. Curr Eye Res 1987;6:127-32.

10. Hara Y, Caspi RR, Wiggert B, Chan C-C, Wilbanks GA, Streilein JW. Suppression of experimental autoimmune uveitis in mice by induction of Anterior Chamber Associated Immune Deviation with interphotoreceptor retinoid binding protein. J Immunol 1992;148:1685-92.

11. Williamson JSP, Bradley D, Streilein JW. Immunoregulatory properties of bone marrow derived cells in the iris and ciliary body. Immunology 1989;67:96-102.

12. McMenamin PG, Hothouse I, Hot PG. Class II major histocompatibility complex (Ia) antigen-bearing dendritic cells within the iris and ciliary body of the rat eye: distribution, phenotype and relation to retinal microglia. Immunology 1992;77:385-93.

13. Wilbanks GA, Mammolenti MM, Streilein JW. Studies on the induction of Anterior Chamber Associated Immune Deviation (ACAID). II. Eye-derived cells participate in generating blood borne signals that induce ACAID. J Immunol 1991;146:3018-24.

14. Streilein JW, Wilbanks GA, Cousins SW. Immunoregulatory mechanisms of the eye. J Neuroimmunol 1002;39:185-200.

15. Knisely TL, Hosoi J, Nazareno R, Granstein R. Aqueous humour contains biologically significant concentrations of glucocorticoids but little or no cortisol binding globulin: relevance to immune privilege in the anterior chamber of the eye. Invest Ophthalmol Vis Sci 1994;35:3711-23.

16. Kaplan HJ, Streilein JW. Immune response to immunisation via the anterior chamber of the eye. I.
$F_{1}$ lymphocyte induced-immune deviation. J Immunol 1977;118:809-14.

17. Bando Y, Ksander BR, Streilein JW. Incomplete activation of lymphokine-producing $\mathrm{T}$ cells by alloantigenic intraocular tumours in ACAID. Immunology 1992;78:266-72.

18. Kosiewicz MM, Okamoto S, Miki S, Ksander BR, Shimizu T, Streilein JW. Imposing deviant immunity on the presensitised state. J Immunol 1994;153:2962-73.

19. Jiang LQ, Jorquera M, Streilein JW. Subretinal space vitreous cavity as immunologically privileged sites for retinal allografts. Invest Ophthalmol Vis Sci 1993;34: 3347-54.

20. Eichorn M, Horneber M, Streilein JW, Lutjen-Drecoll E. Anterior chamber associated immune deviation elicited via primate eyes. Invest Ophthalmol Vis Sci 1993;34:2926-30.

21. Wilbanks GA, Streilein JW. Studies on the induction of Anterior Chamber Associated Immune Deviation (ACAID). I. Evidence that an antigen-specific, ACAID-inducing, cell-associated signal exists in the peripheral blood. J Immunol 1991;146:2610-7.

22. Wilbanks GA, Mammolenti MM, Streilein JW. Studies on the induction of Anterior Chamber-Associated Immune Deviation (ACAID). III. Induction of ACAID depends upon intraocular transforming growth factor- $\beta$. Eur J Immunol 1992;22:165-73.

23. Wilbanks G, Streilein JW. Macrophages capable of inducing anterior chamber associated immune deviation demonstrate spleen-seeking migratory properties. Regional Immunol 1992;4:130-7.

24. Hara Y, Caspi RR, Wiggert B, Dorf M, Streilein JW. Analysis of an in vitro-generated signal that induces systemic immune deviation similar to that elicited by antigen injected into the anterior chamber of the eye. $\mathbf{J}$ Immunol 1992;149:1531-8.

25. Hara Y, Okamoto S, Rouse B, Streilein JW. Evidence that pertioneal exudate cells cultured with eye-derived fluids are the proximate antigen presenting cells in immune deviation of the ocular type. J Immunol 1993;151:5162-71.

26. Okamoto S, Hara Y, Streilein JW. Induction of anterior chamber associated immune deviation (ACAID) with lymphoreticular allogeneic cells. Transplantation 1995; 59:377-81.

27. Niederkorn JY, Streilein JW. Immunogenetic basis for immunologic privilege in the anterior chamber of the eye. Immunogenetics 1981;13:227-36.

28. Jiang LQ, Jorquera M, Streilein JW. Immunologic consequences of intraocular implantation of retinal pigment epithelial allografts. Exp Eye Res 1994; 58:719-28. 